\title{
Early changes in apparent diffusion coefficient as an indicator of response to sorafenib in hepatocellular carcinoma*
}

\author{
Yi-lei ZHAO ${ }^{1}$, Qing-qu GUO ${ }^{\dagger 2}$, Gen-ren YANG ${ }^{1}$, Qi-dong WANG ${ }^{1}$

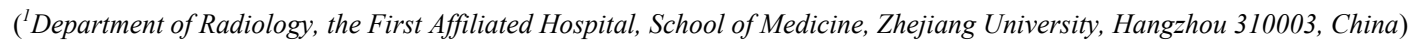 \\ ( ${ }^{2}$ Department of Surgery, the Second Affiliated Hospital, School of Medicine, Zhejiang University, Hangzhou 310009, China) \\ 'E-mail: quguoqing1973@163.com
}

Received Jan. 8, 2014; Revision accepted June 9, 2014; Crosschecked July 18, 2014

\begin{abstract}
Objective: The relationship between apparent diffusion coefficient (ADC) and chemotherapy has been established. However, whether ADC could be considered as a measure for monitoring response to sorafenib in hepatocellular carcinoma (HCC) has not been demonstrated. This study was to investigate the ADC changes of advanced $\mathrm{HCC}$ under sorafenib treatment. Methods: Athymic mice with HepG2 xenografts were allocated to two groups: control and sorafenib (40 mg/kg, bid). T2 and diffusion images were acquired at each time point $(0,10,14$, and $18 \mathrm{~d}$ post-therapy). Tumor volume and changes in ADC were calculated. Results: Tumor volumes on Days 10, 14, and 18 after treatment showed significant decreases in the sorafenib-treated group compared with the control. Pretreatment $A D C$ values were not significantly different between the control and treated groups. A slow increase in ADC in the peripheral zone of tumors appeared in the treated group, which was significantly higher compared with the control group on Days 10, 14, and 18. In the central part of tumors on Day 10 after treatment, an increase in ADC appeared in the treated and control groups, the ADC of the control group being significantly lower compared with the treated tumors. From Day 10 to Day 14, the ADC map showed a progressive decrease in the central region of tumors in the treated and control groups. However, this change is more significant in the treated groups. Conclusions: Early changes in mean ADC correlated with sorafenib treatment in $\mathrm{HCC}$, which are promising indicators for predicting sorafenib response in this carcinoma.
\end{abstract}

Key words: Hepatocellular carcinoma, Sorafenib, Apparent diffusion coefficient, Magnetic resonance imaging (MRI) doi: 10.1631 jzus.B1400010

Document code: A

CLC number: R735.7

\section{Introduction}

Hepatocellular carcinoma (HCC) is becoming more prevalent not only in Southeast Asia and Africa but also in western countries (Kudo, 2008). Of all patients, $30 \%$ to $40 \%$ are diagnosed in the early stages and can be given potentially curative treatments, such as surgical resection, liver transplantation, and ra-

\footnotetext{
* Corresponding author

* Project supported by the National Natural Science Foundation of China (No. 81071960), the New Teacher Foundation of the Ministry of Education, China (No. 20100101120129), and the Zhejiang Provincial Medical Science Research Foundation (No. 2010KYA064), China (c) Zhejiang University and Springer-Verlag Berlin Heidelberg 2014
}

diofrequency ablation; on the basis of early diagnosis and treatment, five-year survival rates of up to $60 \%$ to $70 \%$ can be achieved in early stage patients (Llovet et al., 2003). However, patients with unresectable tumor or metastatic disease have a poor prognosis, reflecting the lack of effective treatment options (Bruix and Sherman, 2005).

Sorafenib, an oral multikinase inhibitor, impedes tumor angiogenesis. Also, it has been shown to increase tumor-cell apoptosis in several tumor models including HCC (Wilhelm et al., 2004). Single-agent sorafenib might have a beneficial therapeutic effect in advanced HCC, prolonging median survival (Llovet et al., 2008). However, once a treatment regimen has 
been designed, close monitoring of tumor response to therapy becomes important; the reason for this is that early markers of therapy response provide key guidelines for oncologists to adjust therapeutic planning during the course of treatment, giving a potential for improved survival for these patients (Seierstad $e t$ al., 2007).

Several magnetic resonance imaging (MRI) modalities have been employed for the early detection of therapy response in various cancer models. In particular, diffusion-weighted MRI (DW-MRI) is becoming increasingly important in the assessment of therapy response. The apparent diffusion coefficient (ADC), reflecting diffusion of water in tissue, can be measured by DW-MRI. Several experimental studies have been completed to probe the predictive value of early changes in ADC using chemotherapy (Thoeny et al., 2005; Seierstad et al., 2007). In this study, our purpose was to evaluate ADC for its ability to monitor $\mathrm{HCC}$ response in athymic mice after administration of sorafenib.

\section{Materials and methods}

\subsection{Cell culture and reagents}

The human HCC cell line HepG2 was purchased from the American Type Culture Collection (ATCC, Rockville, MD, USA). HepG2 cells were maintained in Dulbecco's modified Eagle's medium (DMEM) supplemented with $10 \%$ fetal bovine serum, $100 \mathrm{U} / \mathrm{ml}$ penicillin, and $100 \mathrm{mg} / \mathrm{ml}$ streptomycin in a humidified incubator containing $5 \% \mathrm{CO}_{2}$ in air at $37{ }^{\circ} \mathrm{C}$. Sorafenib (Nexavar ${ }^{\circledR}$ ) was purchased from Bayer Pharmaceuticals (West Haven, CT, USA).

\subsection{Animal experimentation}

Male athymic nu/nu mice, 4-6 weeks old, were obtained from the Zhejiang Chinese Medical University (China) for tumor implantation. All animals were maintained in a sterile environment according to laboratory animal regulations of the Ministry of Science and Technology of the People's Republic of China. The food, water, and bedding for these immunocompromised mice were sterilized and changed at least once a week. A total of $7 \times 10^{6}$ HepG2 cells collected in $100 \mathrm{ml}$ serum-free DMEM in log-phase growth were injected subcutaneously in the backs of mice weighing $20 \mathrm{~g}$. Tumor tissue fragments $\left(2-3 \mathrm{~mm}^{3}\right)$ of human HCC (HepG2) were implanted subcutaneously in the flank region. Once tumor masses became established and palpable, animals were randomized to receive oral gavage of vehicle $(0.9 \%$ sodium chloride $)$ and sorafenib $(40 \mathrm{mg} / \mathrm{kg})$ twice each day. Tumor volumes and body weights were measured weekly. Tumor volume $(V)$ was measured from the longest orthogonal axes $(l)$ and calculated using the formula: $V=l d^{2} / 2$, where width $(d)$ was the shortest measurement. At each time point $(0,10,14$, and $18 \mathrm{~d}$ after treatment), 10 mice ( 5 of each group) were subjected to MRI examination. Animals were anesthetized with a subcutaneous injection of a mixture of $0.5 \%$ pentobarbital, given as a dose of $150 \mu \mathrm{l}$ before MRI examination.

\subsection{MRI examination}

All experiments were performed on a GE Signa HDxt 3.0T clinical whole-body scanner (GE Medical Systems, Milwaukee, WI, USA). The anesthetized mouse was placed in a customized cradle in the supine position, and a high-resolution, dedicated mouse coil was used for signal detection.

All MRI examinations consisted of axial spin echo T1-weighted images (T1WI), fast spin echo T2-weighted images (T2WI), and single shot DWmagnetic resonance (MR) images. Typical acquisition parameters were: T1WI, time of repetition $(\mathrm{TR})=500 \mathrm{~ms}$ and time of echo $(\mathrm{TE})=14 \mathrm{~ms}$; T2WI, $\mathrm{TR}=2000 \mathrm{~ms}$ and $\mathrm{TE}=85 \mathrm{~ms}$; number of excitations $(\mathrm{NEX})=4$; matrix size $=160 \times 128$; FOV $=40 \mathrm{~mm} \times$ $40 \mathrm{~mm}$; slice thickness $=2.00 \mathrm{~mm}$; and, intersection gap $=0.2 \mathrm{~mm}$.

DW echo-planar images were obtained using the following gradient factors: 0 and $500 \mathrm{~s} / \mathrm{mm}^{2}$. The following parameters were used for this sequence: TR/TE, $3300 \mathrm{~ms} / 124 \mathrm{~ms}$; matrix, $64 \times 96$; NEX=8; and, time of acquisition, 2 min $35 \mathrm{~s}$. ADC maps were calculated, and ADC values of region of interest (ROI) were measured in $\mathrm{mm}^{2} / \mathrm{s}$.

\subsection{Image analysis}

The image analysis was performed offline with Functool software at the workstation. The signal intensities of tumors were visually compared with the muscle and graded as hypointense, isointense, or hyperintense. In the transverse T2WI, entire tumors 
were manually delineated on each section with the consensus of two observers. Merging the delineations yielded three-dimensional ROIs of each tumor, based on which the system automatically calculated the volume of each tumor. ROIs of the T2WI were copied to the ADC maps at each time point and manually adjusted for minor distortions that are sometimes visible on ADC maps and are caused by the echo-planar imaging sequence used. ADC values were acquired in the central area and the peripheral zone of each tumor. One circular ROI was placed in the central area (defined as more than $7 \mathrm{~mm}$ from the rim) and four circular ROIs in the peripheral zone (defined as an outer-ring of $5 \mathrm{~mm}$ ). ADC values of the tumor peripheral zone were the average of more than four ROIs. For comparison purposes, a circular ROI in the corresponding location of the contralateral muscles was drawn on a single section.

\subsection{Histologic sections and correlation analysis with MRI}

After the MRI examination, the mice were sacrificed by cervical dislocation. The tumors were immediately excised and fixed in $10 \%$ formalin solution overnight. The tumors were embedded in paraffin, and $4 \mu \mathrm{m}$-thick sections were stained with hematoxylin and eosin. All sections were examined by two pathologists.

\subsection{Statistical analysis}

Data were expressed as mean \pm standard error (SE) and analyzed by a two-tailed $t$-test with $P<0.05$ considered significant. Analyses were performed using SPSS 15.0 statistical software package (SPSS Inc., Chicago, IL, USA).

\section{Results}

\subsection{Effect of sorafenib on tumor growth}

We examined the effects of sorafenib on the growth of subcutaneous xenograft-implanted HCC tumors. HepG2 xenografts were established in athymic mice within 2 weeks and subjected to treatment with sorafenib. The mean tumor volumes of the control and sorafenib-treated mice are shown in Fig. 1. The mean tumor volumes for all groups were similar before sorafenib treatment. We found that the tumor volumes on Days 10, 14, and 18 after the start of treatment showed significant decreases in the sorafenib-treated group compared with the control $(P<0.05)$ (Fig. 1). Importantly, on Days 10 and 18 after administration of sorafenib, tumor growth in the sorafenib-treated group was slower and even arrested compared with Days 0 and 14.

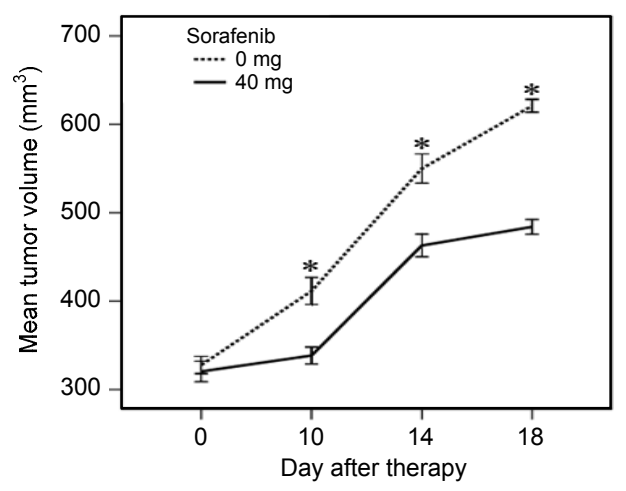

Fig. 1 Effect of sorafenib therapy on mean tumor volumes in hepatocellular carcinoma (HCC)

${ }^{*} P<0.05$, vs. the treated group

\subsection{Basic MRI of tumors}

The T1WI showed homogeneous, low-signal intensity of the entire tumor before sorafenib therapy. The corresponding T2WI revealed a slightly inhomogeneous high-signal intensity. After administration of sorafenib at $10 \mathrm{~d}$, a slightly increased signal intensity in the center of the tumors was shown on T1WI, while a high-signal intensity was observed in the central part of the tumors on T2WI. Nevertheless, the peripheral zone of tumors gave homogeneous signals on T1WI and T2WI. On Days 14 and 18 after administration of sorafenib, a dramatically decreased T1-weighted signal was revealed in the central part of the most of the tumors. Meanwhile, a significantly increased T2-weighed signal was observed in the central part.

\subsection{Effect of sorafenib on ADC}

The mean water ADC values for the control and treated tumors before and after sorafenib treatment are shown in Fig. 2. ADC maps of representative tumors before and after sorafenib-treatment are shown in Fig. 3. Pretreatment ADC values were not significantly different between the control and treated groups. In the control group, the mean ADC of the 


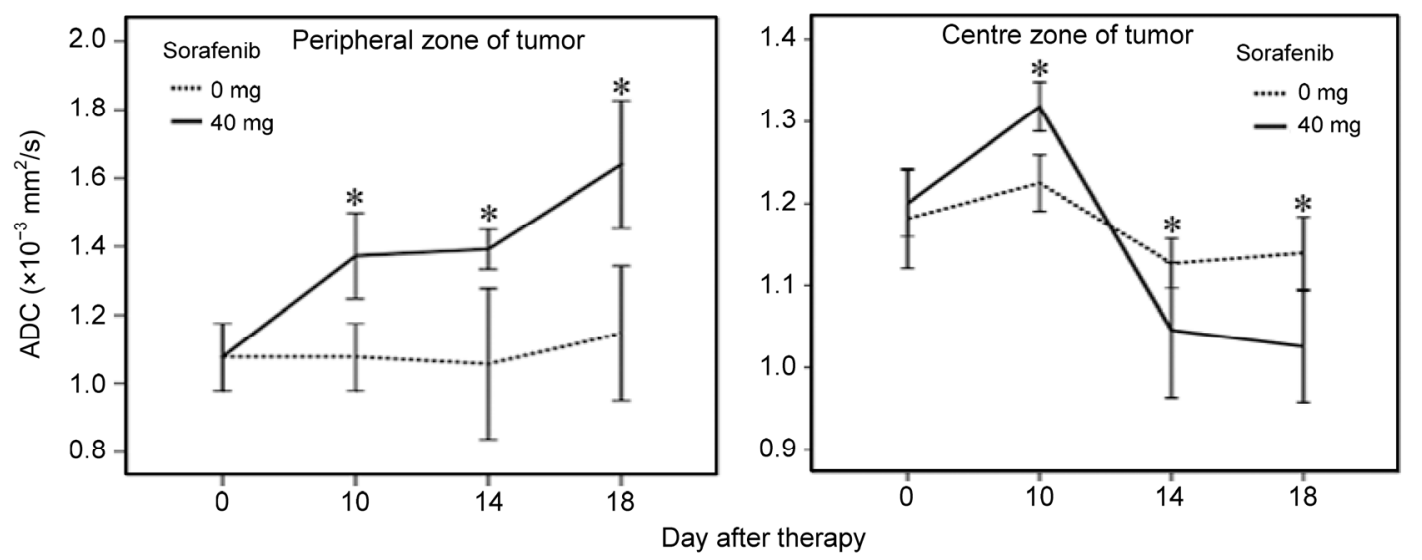

Fig. 2 Effect of sorafenib therapy on the mean water apparent diffusion coefficient (ADC) in hepatocellular carcinoma (HCC)

${ }^{*} P<0.05$, vs. the control group
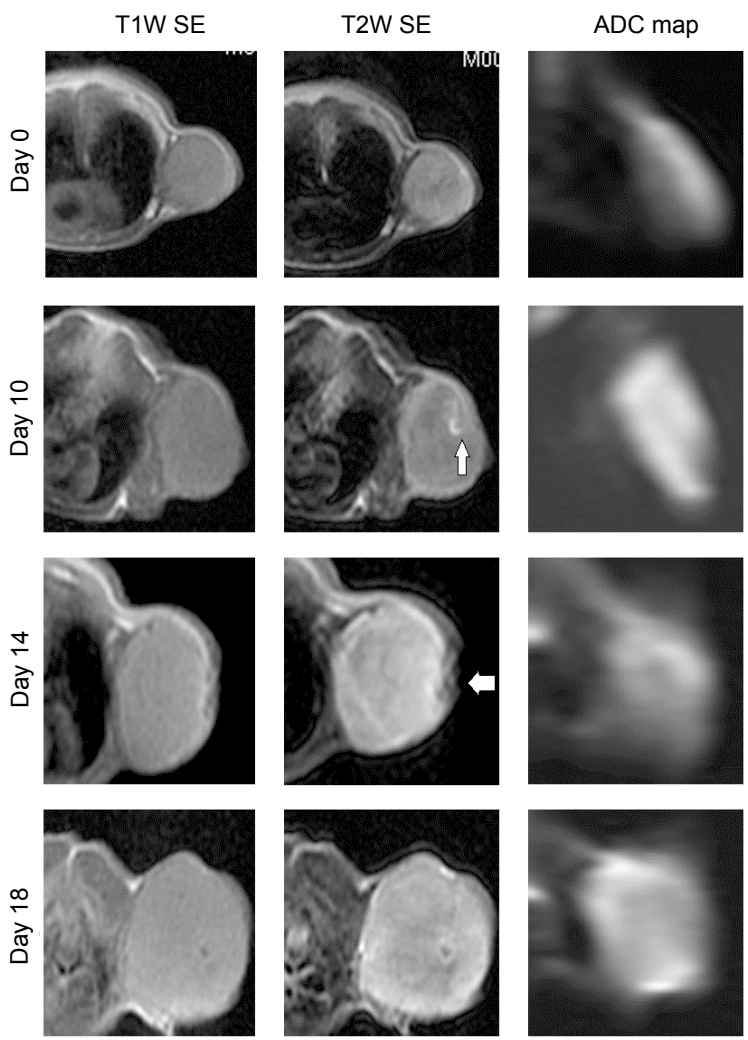

Fig. 3 Water ADC maps of representative treated groups obtained before therapy and at different times post-therapy

At $10 \mathrm{~d}$ after administration of sorafenib, a high-signal intensity was observed in the central part of the tumors on T2-weighted (T2W) images, suggesting necrosis in the central region of tumors (white arrow). At $14 \mathrm{~d}$ after administration, a significant increase in $\mathrm{T} 2 \mathrm{~W}$ images and ulceration of the tumor surface was found (white arrow) peripheral zone did not change significantly in the test process. However, a slow increase of ADC in the peripheral zone of tumors appeared in the treated group, which was significantly higher compared with the control group on Days 10, 14, and $18(P<0.05)$.

In the central region of tumors on Day 10 after treatment, however, an increase in ADC values appeared in the treated and control groups. The ADC values of the control group were significantly lower compared with the values of the treated tumors $(P<0.05)$. Unexpectedly, from Day 10 to Day 14 , the ADC values showed a progressive decrease in the central part of the tumors of the treated and control groups. However, this change was more significant in the treated group compared with the control group $(P<0.05)$.

\subsection{Histology of tumors}

The histology of representative tumors from Days $0,10,14$, and 18 in the treated groups is shown in Fig. 4. Histologic examination of the control group showed no noticeable change throughout the study. However, at $10 \mathrm{~d}$ after sorafenib therapy, histologic analysis showed obvious central necrosis surrounded by a thick peripheral zone of viable tumor cells, where vascular collapse (Fig. 4f) and pronounced edema were found. From Day 14 to Day 18, histologic specimens showed increasingly extensive central necrosis. In addition, many microthrombi appeared in the peripheral region (Fig. 4g) and central part (Fig. 4h) of the tumors. 


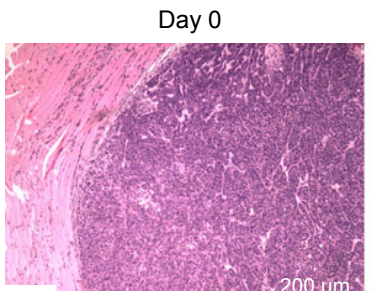

(a)

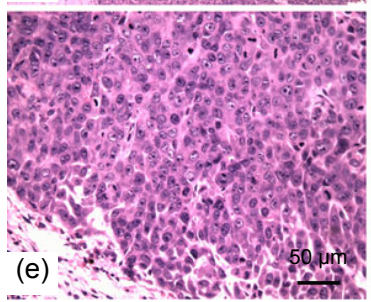

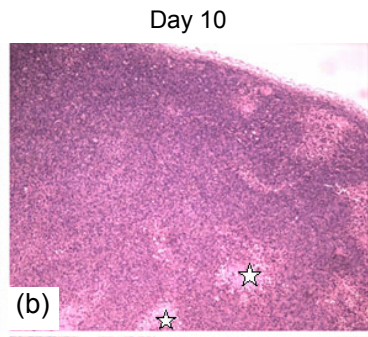

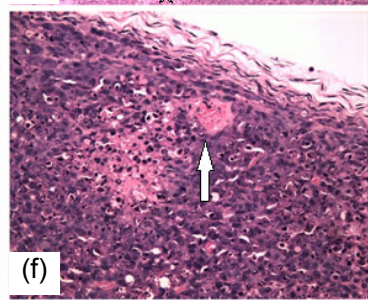

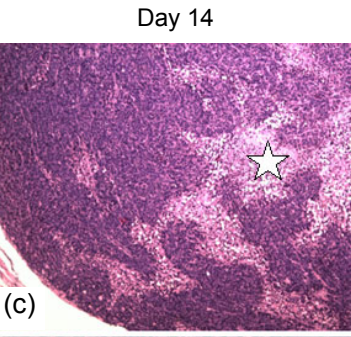

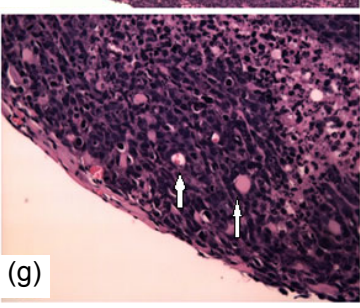

Day 18
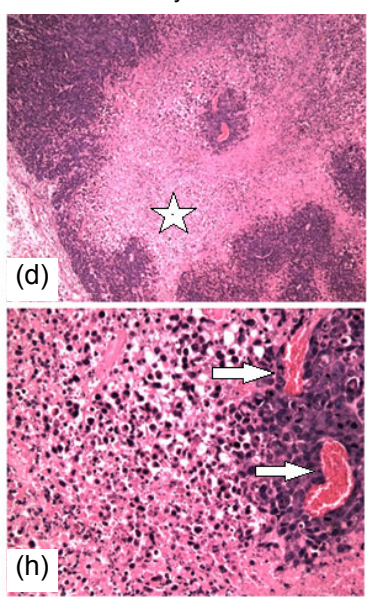

Fig. 4 Hematoxylin and eosin $(\mathrm{H} \& \mathrm{E})$-stained histologic sections of representative treated groups obtained before therapy and at different times post-therapy

(a, e) Day 0; (b, f) Day 10; (c, g) Day 14; (d, h) Day 18. (a-d) Scale bar (white) $200 \mu \mathrm{m}$; (e-h) Scale bar (black) $50 \mu \mathrm{m}$. (b-d) The necrotic regions have become increasingly clear (asterisks); (f) Vascular hyaline degeneration on Day 10 (white arrow); (g) Vascular dilation and microthrombosis in the peripheral region on Day 14 (white arrow); (h) Vascular thrombosis in the central part on Day 18 (white arrow)

\section{Discussion}

As a vascular targeting agent, sorafenib is the first and so far only medication for treating HCC in the clinic. Therefore, it is important to evaluate therapeutic response during HCC therapy. However, conventional anatomical methods of response evaluation, i.e., assessment of lesion size on computer tomography (CT) or MRI, are believed to be of limited value to assess the efficacy of this targeted therapy (Heijmen et al., 2012). For this reason, monitoring intratumoral changes after treatment is an attractive tool for this purpose and may allow an early prediction of treatment response.

Diffusion is a physical property describing the random movement of molecules in response to thermal energy and may be affected by the biophysical properties of tissues such as cell organization and density, microstructure, and microcirculation (Low and Gurney, 2007). For this reason, DW imaging in a number of recent preclinical animal and cell models has proven successful in detecting tumor-cell death following treatment and monitoring or predicting chemotherapeutic response (Moffat et al., 2004; Armitage et al., 2007). The ADC, a value that describes water diffusibility in the presence of factors that restrict diffusion within tissues (e.g., cell membranes, viscosity) (Provenzale et al., 2006), provides a quantifiable measure of the signal and the molecular motion of water in DW-MRI.

Changes in water ADC following chemotherapy have been inconsistently documented. An increase in water ADC after effective chemotherapy has been demonstrated in glioma (Ross et al., 2003; Hall et al., 2004), fibrosarcoma (Zhao et al., 1996), and human breast carcinoma (Galons et al., 1999). By contrast, no significant changes in water ADC were observed in a human breast carcinoma xenograft model after tumor necrosis factor-related apoptosis-inducing ligand therapy despite significant tumor growth delay (Chinnaiyan et al., 2000). However, Dzik-Jurasz et al. reported a decrease in water ADC for some patients with rectal adenocarcinoma after neoadjuvant chemoradiotherapy (Dzik-Jurasz et al., 2002). ADC is linked to tumor pathophysiology in that an increase in water ADC after treatment is related to cell lysis, apoptosis, and necrosis, characterised by cell shrinkage, and nuclear blebbing followed by phagocytosis. On the other hand, a decrease in tumor water ADC is considered to be related to reduced tumor blood flow resulting in focal ischemia/hypoxia and drainage of tumor water (Moffat et al., 2005). It has been 
concluded that a significant increase or decrease in tumor ADC values occurring after treatment indicates the likelihood of a response to therapy.

In this study, our principal aim was to determine whether non-invasive measurement of tumor ADC via MRI can detect early changes in tumor pathophysiology in response to sorafenib treatment. From the results, we can see that the tumor volume of the treatment group was clearly reduced compared with the control group on Days 10, 14, and 18. In DW-MRI, a slow increase in ADC in the peripheral zone of tumors was apparent in the treated group during the same period, which was significantly higher compared with the control group. In the central part of tumors on Day 10, a similar increase in ADC was apparent in the treated and control groups. Nevertheless, on Day 14, the ADC map showed a progressive decrease in the central part of the treated and control groups. However, this change is more significant in the treated group than in the control group. From the results in our study, we believe sorafenibinduced changes in HCC pathophysiology altered tumor water ADC. The marked changes of ADC during the different periods of treatment reflected pathophysiological intracellular changes.

In the early stages of therapy, ADC values increased in both the central area and peripheral zone. The histologic changes - a necrotic area surrounded by a thick peripheral zone of viable tumor cells featuring vascular collapse and pronounced edema - can be explained as causing high ADC values, in turn resulting from rapid diffusion of water protons due to lost membrane integrity and permeability (Lyng et al., 2000). Interestingly, from Day 10 to Day 18 of sorafenib therapy, microthrombi in small vessels were found in the central part of the tumor. The reduced $\mathrm{ADC}$ values in the central part may be caused by reduced tumor blood flow resulting in focal ischemia/ hypoxia and drainage of tumor water (Moffat et al., 2005). In addition, we observed increased cellular density and vascular dilation in some peripheral regions. The increase in ADC may reflect recovering blood flow in the tumor periphery (Thoeny et al., 2005). According to a previous report, certain antiangiogenic agents can also transiently 'normalize' the abnormal structure and function of tumor vasculature to make it more efficient for oxygen and drug delivery (Jain, 2005). We, therefore, think that vascular nor- malization of abnormal structures in $\mathrm{HCC}$ caused by sorafenib may be due to an ADC increase in the tumor periphery.

One shortcoming of this study is the limited number of animals. Furthermore, earlier time points of sorafenib treatment, for example, Day 1, 2, or 4 after treatment, would give a better statistical result.

In conclusion, these data illustrate that sorafenib is effective in treating HCC. In vivo MRI experiments have shown that early changes in ADC are promising signs for effective sorafenib treatment, indicating that these parameters could yield clinically important information in the prediction of sorafenib response for HCC.

\section{Compliance with ethics guidelines}

Yi-lei ZHAO, Qing-qu GUO, Gen-ren YANG, and Qi-dong WANG declare that they have no conflict of interest. All institutional and national guidelines for the care and use of laboratory animals were followed.

\section{References}

Armitage, P.A., Schwindack, C., Bastin, M.E., et al., 2007. Quantitative assessment of intracranial tumor response to dexamethasone using diffusion, perfusion and permeability magnetic resonance imaging. Magn. Reson. Imaging, 25(3):303-310. [doi:10.1016/j.mri.2006.09.002]

Bruix, J., Sherman, M., 2005. Management of hepatocellular carcinoma. Hepatology, 42(5):1208-1236. [doi:10.1002/ hep.20933]

Chinnaiyan, A.M., Prasad, U., Shankar, S., et al., 2000. Combined effect of tumor necrosis factor-related apoptosisinducing ligand and ionizing radiation in breast cancer therapy. PNAS, 97(4):1754-1759. [doi:10.1073/pnas. 030545097]

Dzik-Jurasz, A., Domenig, C., George, M., et al., 2002. Diffusion MRI for prediction of response of rectal cancer to chemoradiation. Lancet, 360(9329):307-308. [doi:10. 1016/S0140-6736(02)09520-X]

Galons, J.P., Altbach, M.I., Paine-Murrieta, G.D., et al., 1999. Early increases in breast tumor xenograft water mobility in response to paclitaxel therapy detected by non-invasive diffusion magnetic resonance imaging. Neoplasia, 1(2): 113-117. [doi:10.1038/sj.neo.7900009]

Hall, D.E., Moffat, B.A., Stojanovska, J., et al., 2004. Therapeutic efficacy of DTI-015 using diffusion magnetic resonance imaging as an early surrogate marker. Clin. Cancer Res., 10(23):7852-7859. [doi:10.1158/1078-0432. CCR-04-1218]

Heijmen, L., Verstappen, M.C., ter Voert, E.E., et al., 2012. Tumour response prediction by diffusion-weighted MR imaging: ready for clinical use? Crit. Rev. Oncol. Hematol., 83(2):194-207. [doi:10.1016/j.critrevonc.2011.12.008] 
Jain, R.K., 2005. Normalization of tumor vasculature: an emerging concept in antiangiogenic therapy. Science, 307(5706):58-62. [doi:10.1126/science.1104819]

Kudo, M., 2008. Hepatocellular carcinoma 2009 and beyond: from the surveillance to molecular targeted therapy. Oncology, 75(Suppl. 1):1-12. [doi:10.1159/000181865]

Llovet, J.M., Burroughs, A., Bruix, J., 2003. Hepatocellular carcinoma. Lancet, 362(9399):1907-1917. [doi:10.1016/ S0140-6736(03)14964-1]

Llovet, J.M., Ricci, S., Mazzaferro, V., et al., 2008. Sorafenib in advanced hepatocellular carcinoma. N. Engl. J. Med., 359(4):378-390. [doi:10.1056/NEJMoa0708857]

Low, R.N., Gurney, J., 2007. Diffusion-weighted MRI (DWI) in the oncology patient: value of breathhold DWI compared to unenhanced and gadolinium-enhanced MRI. $J$. Magn. Reson. Imaging, 25(4):848-858. [doi:10.1002/ jmri.20864]

Lyng, H., Haraldseth, O., Rofstad, E.K., 2000. Measurement of cell density and necrotic fraction in human melanoma xenografts by diffusion weighted magnetic resonance imaging. Magn. Reson. Med., 43(6):828-836. [doi:10.1002/ 1522-2594(200006)43:6<828::AID-MRM8>3.0.CO;2-P]

Moffat, B.A., Hall, D.E., Stojanovska, J., et al., 2004. Diffusion imaging for evaluation of tumor therapies in preclinical animal models. MAGMA, 17(3-6):249-259. [doi:10.1007/s10334-004-0079-z]

Moffat, B.A., Chenevert, T.L., Lawrence, T.S., et al., 2005. Functional diffusion map: a noninvasive MRI biomarker for early stratification of clinical brain tumor response. $P N A S$, 102(15):5524-5529. [doi:10.1073/pnas.0501532102]

Provenzale, J.M., Mukundan, S., Barboriak, D.P., 2006. Diffusion-weighted and perfusion MR imaging for brain tumor characterization and assessment of treatment response. Radiology, 239(3):632-649. [doi:10.1148/radiol. 2393042031]

Ross, B.D., Moffat, B.A., Lawrence, T.S., et al., 2003. Evaluation of cancer therapy using diffusion magnetic resonance imaging. Mol. Cancer Ther., 2(6):581-587.

Seierstad, T., Folkvord, S., Roe, K., et al., 2007. Early changes in apparent diffusion coefficient predict the quantitative antitumoral activity of capecitabine, oxaliplatin, and irradiation in HT29 xenografts in athymic nude mice. $\mathrm{Ne}$ oplasia, 9(5):392-400. [doi:10.1593/neo.07154]

Thoeny, H.C., de Keyzer, F., Chen, F., et al., 2005. Diffusionweighted MR imaging in monitoring the effect of a vascular targeting agent on rhabdomyosarcoma in rats. $R a-$ diology, 234(3):756-764. [doi:10.1148/radiol.2343031721]

Wilhelm, S.M., Carter, C., Tang, L., et al., 2004. BAY 43-9006 exhibits broad spectrum oral antitumor activity and targets the RAF/MEK/ERK pathway and receptor tyrosine kinases involved in tumor progression and angiogenesis. Cancer Res., 64(19):7099-7109. [doi:10. 1158/0008-5472.CAN-04-1443]

Zhao, M., Pipe, J.G., Bonnett, J., et al., 1996. Early detection of treatment response by diffusion-weighted ${ }^{1} \mathrm{H}-\mathrm{NMR}$ spectroscopy in a murine tumour in vivo. Br. J. Cancer, 73(1):61-64. [doi:10.1038/bjc.1996.11]

\section{中文概要：}

\section{本文题目：磁共振弥散加权成像对索拉菲尼治疗肝细胞癌的疗效评估}

Early changes in apparent diffusion coefficient as an indicator of response to sorafenib in hepatocellular carcinoma

研究目的: 应用磁共振弥散加权成像 (DWI) 监测荷瘤裸鼠肝细胞性肝癌表观弥散系数 (ADC) 的变化, 探讨 ADC 对索拉菲尼治疗肝细胞性肝癌的疗效预测。

创新要点: 探讨磁共振 DWI 对索拉菲尼治疗肝癌疗效的早期评估, 期望能够指导临床合理用药。

研究方法: 荷瘤裸鼠分成两组（治疗组和对照组）共 40 只, 使用 GE 3.0T 磁共振成像系统和小动物线 圈, 分别于服药前后 $1 、 10 、 14$ 和 18 天 ( $40 \mathrm{mg} / \mathrm{kg}$, 一天两次) 进行 $\mathrm{T} 1$ 加权成像（T1WI）、 $\mathrm{T} 2$ 加权成像（T2WI）和 DWI 扫描。在弥散加权图像上进行 ADC 测量, 对比不同区域和不 同时间点的差异。

重要结论: 索拉菲尼治疗 10 天后, 伴随肝癌肿瘤的缩小, 肿瘤内部 ADC 值明显上升, 病理切片显示治 疗组瘤体中心小血管闭塞, 调亡细胞可见, 此时瘤体内坏死细胞并未大量显现, 说明 ADC 值的改变可以反映索拉菲尼的疗效。

关键词组: 肝细胞癌; 索拉菲尼; 表观弥散系数; 磁共振成像 